\title{
Hybrid image visualization tool for 3D integration of CT coronary anatomy and quantitative myocardial perfusion PET
}

\author{
Martina Marinelli • Vincenzo Positano - Stephan G. Nekolla • Paolo Marcheschi • \\ Giancarlo Todiere - Natalia Esposito • Stefano Puzzuoli • Giuseppe A. L'Abbate • \\ Paolo Marraccini · Danilo Neglia
}

Received: 10 January 2012 / Accepted: 11 June 2012 / Published online: 3 July 2012

(c) CARS 2012

\begin{abstract}
Purpose Multimodal cardiac imaging by CTA and quantitative PET enables acquisition of patient-specific coronary anatomy and absolute myocardial perfusion at rest and during stress. In the clinical setting, integration of this information is performed visually or using coronary arteries distribution models. We developed a new tool for CTA and quantitative PET integrated 3D visualization, exploiting XML and DICOM clinical standards.

Methods The hybrid image tool (HIT) developed in the present study included four main modules: (1) volumetric registration for spatial matching of CTA and PET data sets, (2) an interface to PET quantitative analysis software, (3) a derived DICOM generator able to build DICOM data set from quantitative polar maps, and (4) a 3D visualization tool of integrated anatomical and quantitative flow information. The four modules incorporated in the HIT tool communicate by defined standard XML files: XML-transformation and XML MIST standards.

Results The HIT tool implements a 3D representation of CTA showing real coronary anatomy fused to PET-derived quantitative myocardial blood flow distribution. The technique was validated on 16 data sets from EVINCI study pop-
\end{abstract}

M. Marinelli $(\varangle) \cdot$ N. Esposito · S. Puzzuoli · P. Marraccini ·

D. Neglia

Institute of Clinical Physiology, CNR, Via Moruzzi n.1,

56124 Pisa, Italy

e-mail: martina.marinelli@ifc.cnr.it

V. Positano · P. Marcheschi · G. Todiere · G. A. L'Abbate ·

D. Neglia

Fondazione Gabriele Monasterio, CNR-Regione Toscana,

Via Moruzzi n.1, 56124 Pisa, Italy

S. G. Nekolla

Nuklearmedinische Klinik der TU München, Ismaningerstr. 22,

81675 Munich, Germany ulation. The validation of the method confirmed the high matching between "original" and derived data sets as well as the accuracy of the registration procedure.

Conclusions Three-dimensional integration of patientspecific coronary artery anatomy provided by CTA and quantitative myocardial blood flow obtained from PET imaging can improve cardiac disease assessment. The HIT tool introduced in this paper may represent a significant advancement in the clinical use of this multimodal approach.

Keywords CAD · Hybrid imaging . Myocardial flow reserve $\cdot \mathrm{XML} \cdot 3 \mathrm{D}$ visualization

\section{Introduction}

The integration of myocardial perfusion information, as imaged by positron emission tomography (PET), and coronary artery anatomy, obtained using computed tomography angiography (CTA) technology, is an useful and validated method to improve the noninvasive diagnosis of coronary artery disease (CAD) [1-5]. Indeed, the multimodal integration of CTA and PET imaging gives the opportunity to combine complementary data such as the anatomical degree of stenosis with the regional information on functional defects, under both rest and stress conditions, improving the ability to characterize the functional consequences of anatomical pathology onto myocardial perfusion [6]. The merged visualization of complementary information is based on the accurate temporal and spatial registration of the two involved data sets. This goal can potentially be achieved using the hybrid PET/CT tomographs that are capable to collect multimodal cardiac data using a single-session acquisition protocol with sequential CTA and PET imaging without the patient to be drawn out from the scanner. In this way, the misalignment 
CTA
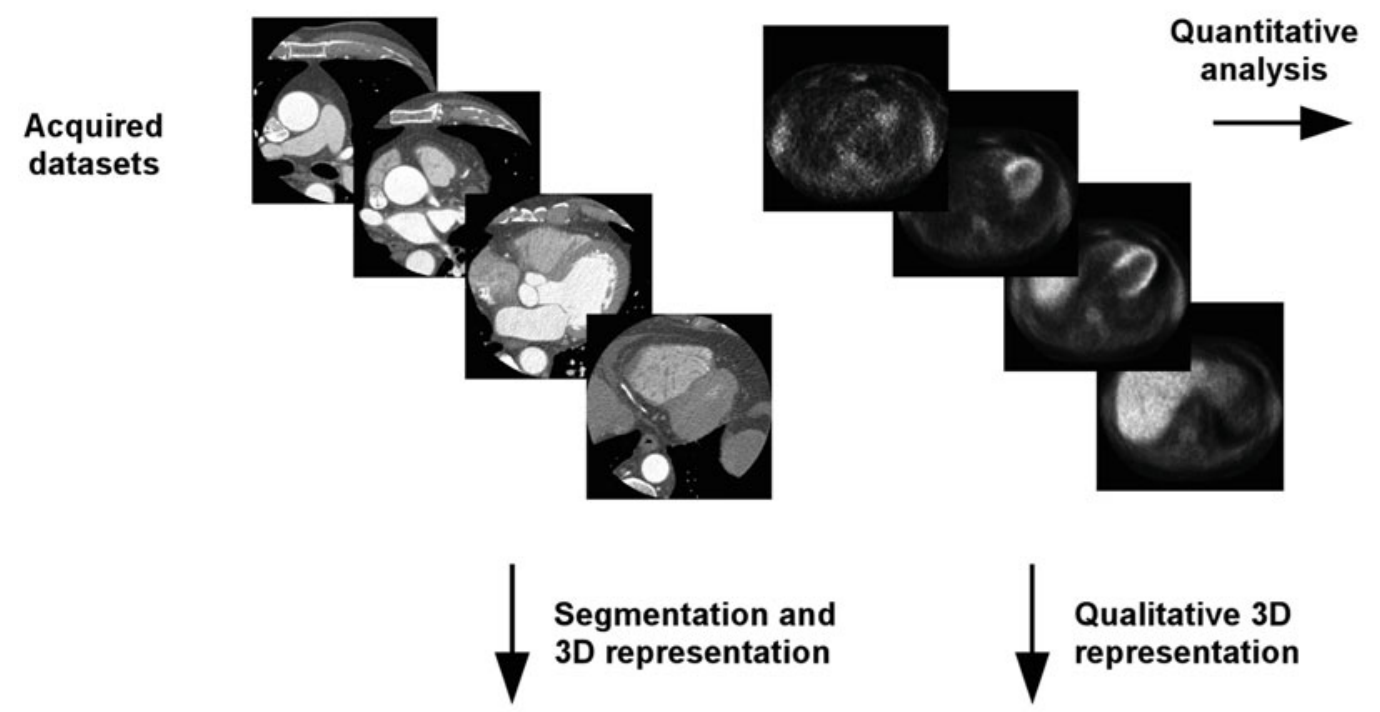

PET

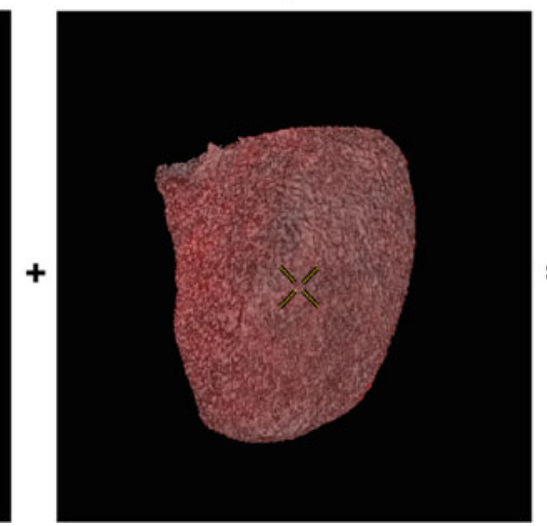

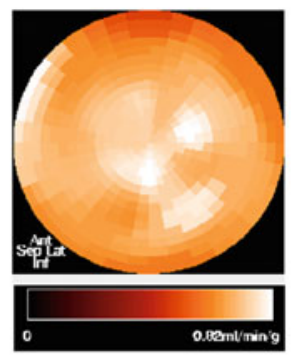

CTA + PET

Fused 3D view

\section{Reconstructed datasets}

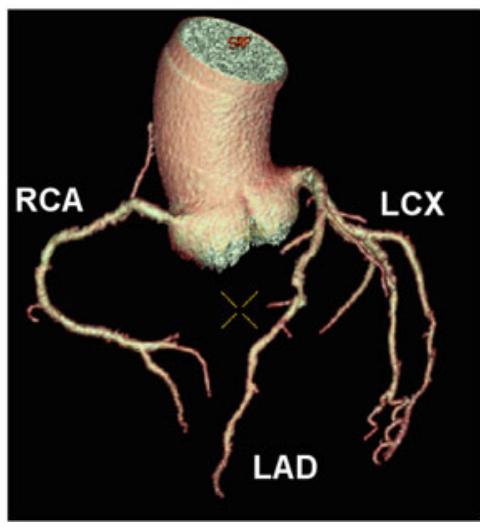

Fig. 1 Example of three-dimensional fusion of CTA and "raw" PET obtained using the GE AWS-2.0-5.0 (Advantage Workastation ${ }^{\mathrm{TM}}$ Server). The processing of CTA and PET images produces a $3 \mathrm{D}$ visu- alization of coronary arteries anatomy and qualitative myocardial perfusion, respectively. The 3D fused representation is also obtained. The quantitative analysis of PET data produces a polar map visualization between the two cardiac data sets is reduced, even if it is not completely solved due to the patient's movement and breathing during the examination. For this reason, even for single-session hybrid scanner, an alignment correction could be necessary [7]. Alternatively to this approach, a multi-session acquisition protocol can be considered using either a hybrid scanner or separate devices. In this case, the integration of complementary information by software techniques [8] is always required to match the cardiac data sets.

Independently on the method used to align the image data, a multistage image analysis protocol should be used to obtain the relevant clinical information. As shown in Fig. 1, the acquired CTA data set is segmented and visualized in 3D to allow diagnosis and localization of coronary stenosis. The PET data set is segmented and visualized in $3 \mathrm{D}$ as well, leading to a representation of the "raw" PET signal that assesses semiquantitatively the myocardial perfusion.

Several commercial software tools used in clinical routine permit to import the PET and CTA cardiac data sets, eventually adjusting the matching if needed, and allow to obtain the fused and three-dimensional visualization of real coronary artery anatomy and semiquantitative myocardial perfusion function. This fused visualization has an added value for CAD assessment, giving the possibility to overcome the limitation imposed by the fixed assignment of left ventricle myocardial segments to the vascular territories (left anterior descending coronary artery: LAD; left circumflex: LCX; right coronary artery RCA) following the American Heart Association (AHA) standard segmentation [4,9]. Available software tools for registration and fusion application utilize semiquantitative PET data sets. It means that a regional 

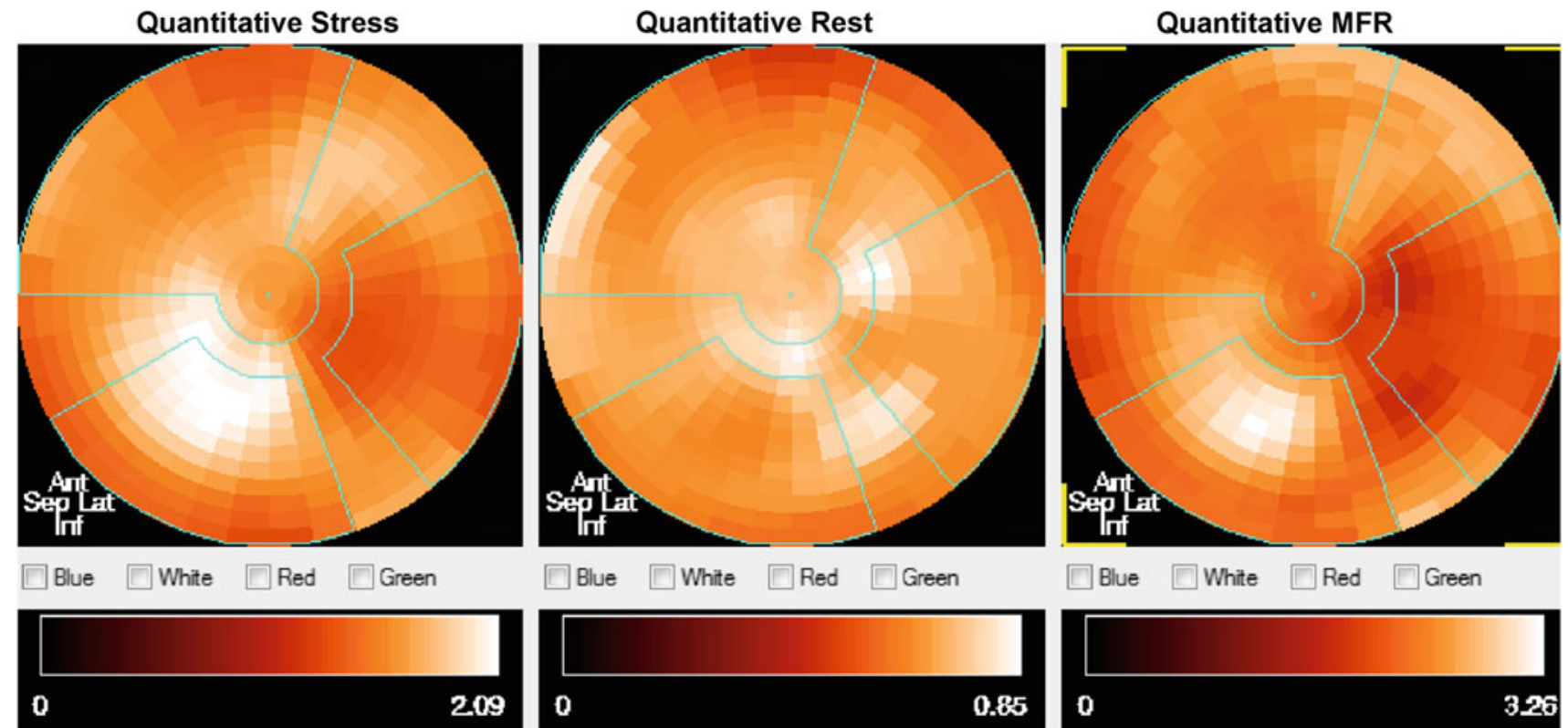

$\square$ Blue $\square$ White $\square$ Red $\square$ Green
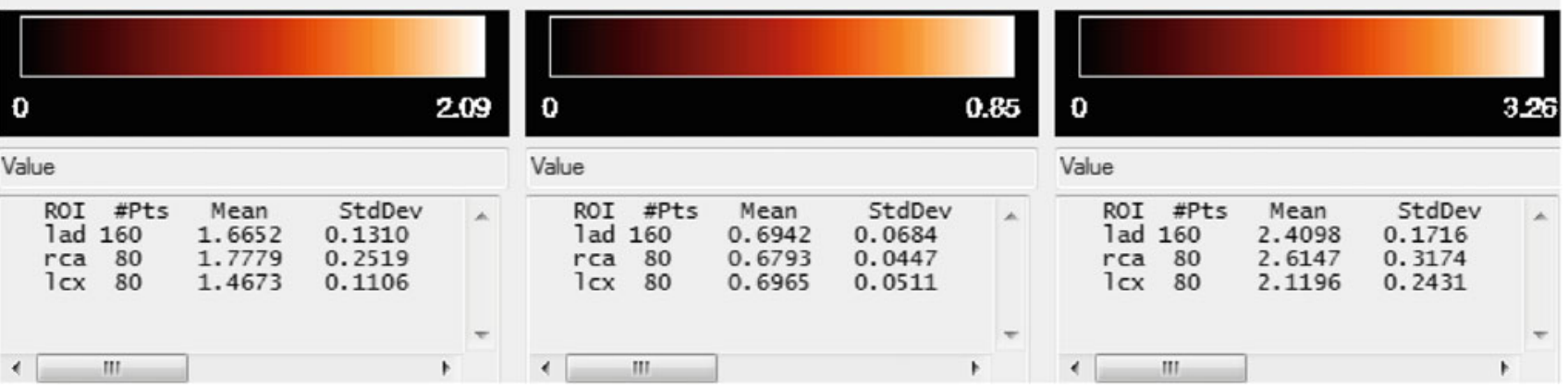

Value

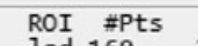

ROI \#Pts
lad 160

rca 80

2.4098
2.6147

2.6147
2.1196

StdDev

0.1716

0.3174

0.2431

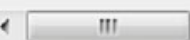

Fig. 2 Example of typical output of the software used for the quantitative analysis of PET data. The stress, rest, and myocardial flow reserve (stress/rest values) polar maps are segmented using the AHA standard segmentation. The MunichHeart software has been used for quantitative analysis of PET data reduction in myocardial perfusion will be well visualized, while a global reduction in perfusion that could be associated with several cardiac diseases as balanced three vessel disease or dilatative cardiomyopathy [10] might be not detected.

Luckily enough, PET imaging allows quantitative measurement of myocardial blood flow. Indeed, from the dynamic PET data sets, absolute $(\mathrm{ml} / \mathrm{min} / \mathrm{g}$ ) myocardial blood flow at rest and during stress can be determined by appropriate modeling $[6,11,12]$, and myocardial flow reserve (MFR) can be computed as the ratio between the stress and rest quantitative values. Quantitative perfusion evaluation is generally performed by a dedicated image analysis tools that provide a polar map representation (i.e., bull's eye) of absolute flow and MFR, following AHA guidelines [9]. Figure 2 shows the typical output of the PET quantitative analysis. It is important to note that in this representation, the relationship between coronary anatomy and myocardial perfusion is defined following AHA standardization and cannot be referred to the patient-specific coronary anatomy provided by CTA.

Accordingly, while the assessment of CAD and its functional effects on myocardial perfusion could be definitely improved by using 3D integration and visualization of quantitative cardiac perfusion and coronary anatomy, available software tools do not allow this representation. Stolzmann et al. [13] demonstrated the added clinical value of combined CTA anatomy with magnetic resonance (MR) cardiac perfusion imaging. However, the 3D integrated visualization of real coronary artery anatomy with quantitative myocardial blood flow from PET imaging, still considered the gold-standard of absolute perfusion, has higher potential. An example of such integration for CAD evaluation is described by Kajander et al. [14] and Knuuti et al. [15] where an absolute scale was used to obtain the standard display of myocardial perfusion information.

The purpose of this paper is to describe a new method (hybrid image tool-HIT) developed to integrate the anatomical and functional quantitative data coming from CTA and PET, respectively. The aim of this method is to obtain the 3D fused visualization of real coronary anatomy and quantitative myocardial perfusion stress, myocardial perfusion rest, and myocardial flow reserve for the accurate evaluation of cardiac diseases. 
Fig. 3 Schema of the developed Hybrid Image Tool

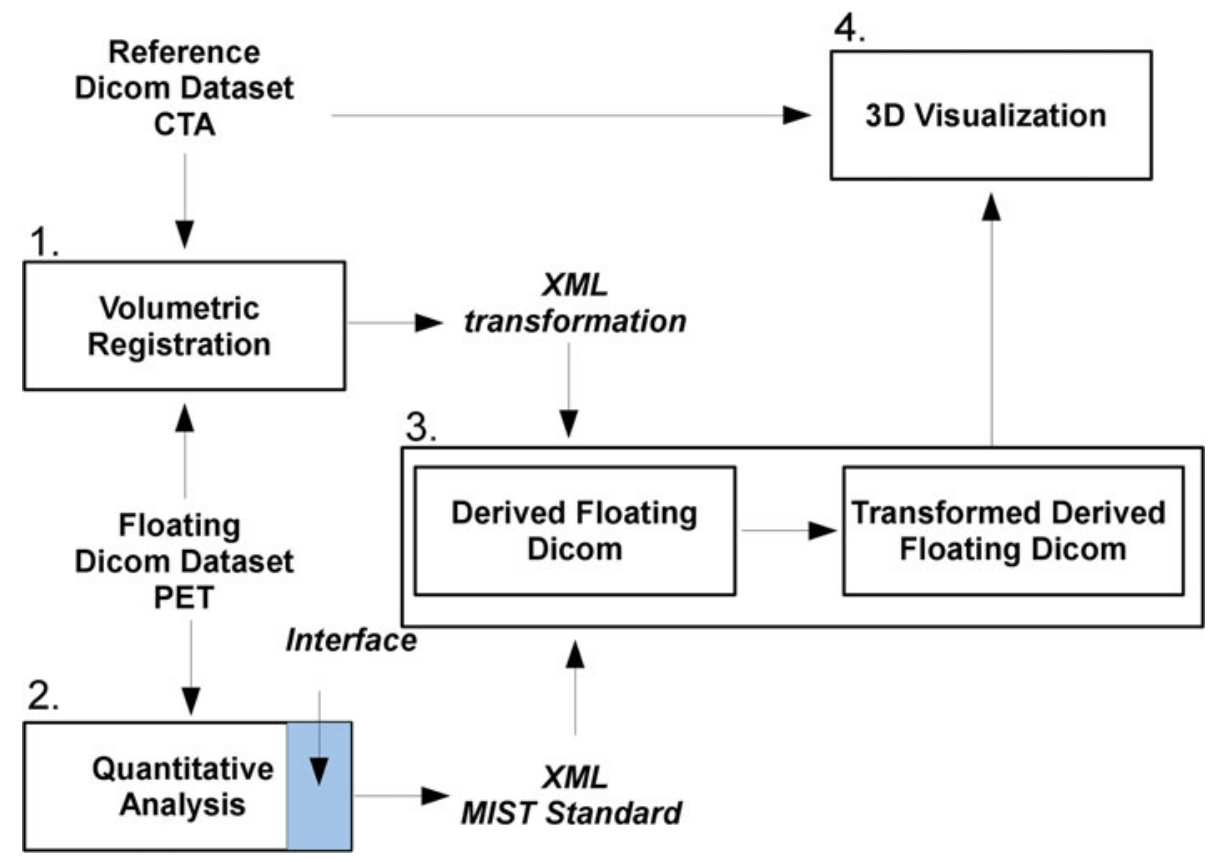

\section{Material and methods}

Population and image acquisition

Eight patients randomly extract from EVINCI study population underwent a multimodal imaging protocol consisted of consecutive acquisitions of cardiac PET at rest and during stress for the assessment of MFR and CTA for the visual evaluation of coronary anatomy. Both PET and CTA acquisitions were performed using a PET/CT device (Discovery VCT, GE Healthcare, Milwaukee, WisconsinUSA).

The PET data sets were acquired after intravenous injection of ${ }^{13} \mathrm{NH}_{3}(7.4 \mathrm{MBq} / \mathrm{kg}$ body weight $-0.2 \mathrm{mCi} / \mathrm{kg}$, in 20-30s). Dynamic acquisition in 2D-mode was started simultaneously with tracer injection $(20$ frames $\times 6 \mathrm{~s}, 6$ frames $\times 30 \mathrm{~s}$ and 3 frames $\times 300 \mathrm{~s})$, followed by a final static acquisition in 3D-mode $(1$ frame $\times 600 \mathrm{~s})$. After completion of the baseline study (administered activity: $416.17 \pm 49 \mathrm{MBq}$ ) and waiting additional $15 \mathrm{~min}$ to allow for radioactive decay, patients underwent intravenous dipyridamole $(0.56 \mathrm{mg} / \mathrm{Kg}$ in $4 \mathrm{~min})$ stress testing and the ${ }^{13} \mathrm{NH}_{3}$ PET imaging was repeated as previously described (administered activity: $432.33 \pm 103.27 \mathrm{MBq}$ ) [16]. In all cases, the transaxial images (47 slices) of $128 \times 128$ pixels with voxel size $2.73 \mathrm{~mm} \times 2.73 \mathrm{~mm} \times 3.27 \mathrm{~mm}$ were obtained.

In a different day, patients underwent a MSCT coronary angiography with the following scan parameters: retrospective ECG gating; 912 channel detectors along the gantry and 64 channel detectors along the $z$-axis (radiation dose: $14.83 \pm 3.3 \mathrm{mSv}$ ). Nonionic iodinated contrast medium
(Iomeprol 400, Bracco, Italy) was injected and followed by a saline flush via a peripheral vein using a programmable injector. Scans were performed during breath hold. Beta-blocker was used depending on the patient's heart rate. The CTA data set consisted of transaxial images (range 202:235 slices) of $512 \times 512$ pixels with voxel size $0.33 \mathrm{~mm} \times 0.33 \mathrm{~mm} \times$ $0.62 \mathrm{~mm}$.

The study complied with the Declaration of Helsinki. All subjects gave written informed consent to the study.

Hybrid image tool

The acquired PET and CTA cardiac data sets were used as input to the hybrid image tool (HIT) graphically represented in Fig. 3.

The purpose of the developed tool is to allow a multimodal standard storing and transfer of both header DICOM and image information, such as the myocardial geometrical information and the regional quantitative values, with the final goal to reach an accurate 3D integrated visualization and correspondence between coronary anatomy and myocardial function.

Four main modules can be identified on Fig. 3:

1. the volumetric registration tool for spatial matching of reference (anatomical) and floating (functional) data sets;

2. the quantitative analysis of functional data sets using validated software normally used in the clinical routine;

3. the generator of the derived functional data sets; 
4. the $3 \mathrm{D}$ visualization tool of integrated anatomical and quantitative functional information.

The four modules will be discussed in detail below. The communication between the different modules has been established using a common interface based on the standard eXtensible Markup Language (XML) protocol [17]. The development of the whole process, including modules and XML communication interface, was based on the use of IDL (version 8.0, Exelis Visual Information Solutions, Boulder, CO, USA).

\section{Volumetric registration module}

The volumetric registration tool was necessary for the spatial alignment of reference and floating data sets. For the purpose of this paper, the CTA data volume, showing the anatomical cardiac information, has been set as reference data set, and both static stress and rest PET data volume have been used as floating data sets. Two XML files (XML-transformation), containing the rotational and translational matching parameters, were defined in order to store and transfer the result of the performed alignment processes.

\section{Quantitative analysis module}

The acquired functional data sets have been used as input to the quantitative analysis module. The purpose of this module is to create a high resolution quantitative polar map (as shown in Fig. 2) and to save it using a XML file. This standard file has been proposed and created in order to guarantee the multimodal image storage and transfer of quantitative information (XML MIST : XML Multimodal Image Storage and Transfer). For this reason, an "interface" between the quantitative analysis tool and the secondary DICOM generator (Fig. 3) has been developed in order to include into the XML MIST file the high resolution polar map, described by the quantitative values and by the geometrical position of each sector included in the polar map. Figure 4 illustrates the structure of the XML MIST file. A polar map XML root element includes a "General DICOM Information" tag, with the appropriate DICOM header information elements. Each ring of the polar map, corresponding to a short axis PET slice, is codified as a XML tag. For each polar map sector, the geometrical coordinates in the patient's space and the corresponding value of the quantitative perfusion are reported.

The analysis of the stress and rest dynamic PET cardiac data sets produced two XML MIST files. An additional processing of stored data could be also performed in order to calculate the derived quantitative parameter, such as the MFR defined as the ratio between the stress and rest quantitative values. Hence, the XML MIST file containing the derived quantitative values could be also saved.

\section{Derived DICOM module}

The saved XML MIST files have been used as input to the derived DICOM module. The purpose of this module is to create a derived DICOM data set, representing the quantitative polar maps, using the information coming from the saved XML files. As also emphasized by Knuuti et al. [15], the visualization of quantitative perfusion images for clinical purposes should be standardized using a fixed scale. For this reason, a fixed scale was set and used to represent the quantitative DICOM data set. The header DICOM fields related to the visualization were consequently changed. To guarantee the registration between CTA anatomy and quantitative myocardial function data resulting from PET analysis, the rigid transformations stored using the XML-transformation files were applied to the corresponding floating rest and stress quantitative data sets.

\section{$3 D$ visualization}

The transformed quantitative PET volumes and the CTA data set were used as input to the $3 \mathrm{D}$ visualization module. The purpose of this module is to visually integrate the high resolution quantitative functional information to the real coronary anatomy. Any software allowing the three-dimensional segmentation of coronary arteries anatomy and the fusion and visualization with functional data set can be exploited and included in the developed method.

\section{Validation}

Eight CTA and sixteen PET (rest and stress) data sets, acquired using the imaging protocol previously described, were used for HIT validation. Image fusion between derived quantitative PET data sets, obtained using the method described above, and the corresponding "original" PET data sets was performed and the resulting representations were assessed by two expert observers. Each observer evaluated whether a further registration between the two data sets was needed using a two-point scale (1: no registration needed; 2 : registration needed). Moreover, the quality of the matching between derived and "original" data sets was scored by a fivepoint scale (1: not acceptable; 2: suboptimal; 3: acceptable; 4: good; 5: excellent).

A second validation step was also applied. The 3D integration of CTA and original PET (rest and stress) data was obtained by spatial alignment of volumetric data sets obtained by applying the registration method previously described. The quality of the sixteen 3D integrated visualizations was assessed by two expert observers using the previously reported five-point scale. The calculated CTA/original PET spatial transformations were also applied to derive quantitative PET data sets in order to obtain a 3D 


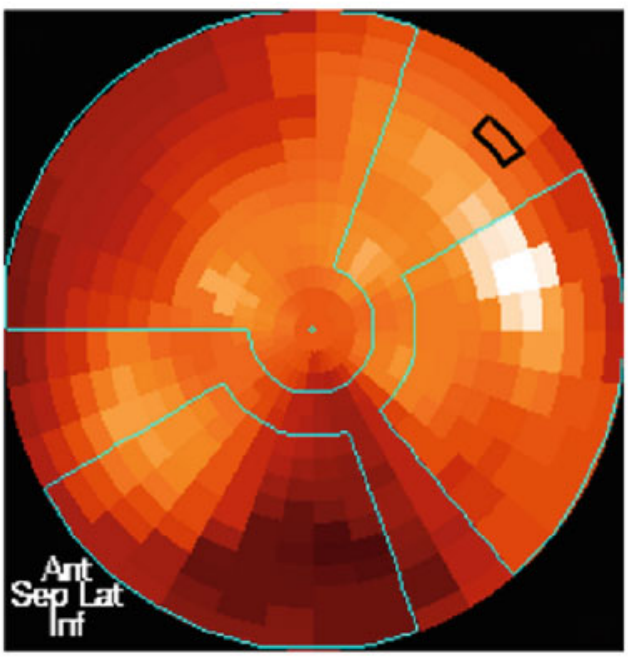

- <Image_Information $>$

$\langle$ Slice_number $>3<$ Slice_number $>$

$<$ Slice_type $>\mathrm{NaN}</$ Slice_type $>$

$<$ Sectors_number $>36<$ Sectors_number $>$

$-<$ Midwall countours>

$95.0315-42.5188-189.221 .95 .8770-40.8930-189.83096 .9197-39.3457-190.23498 .0086-37.7795-190.30999 .1106-36.2419-190.052100 .192 \quad \cdots$

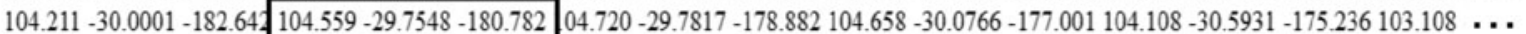
$98.4653-37.1993-169.39397 .6811-38.7827-169.20297 .0616-40.5651-169.19496 .4279-42.3841-169.50095 .6187-43.9548-170.24994 .6996$. . . $92.1273-47.6499-179.92892 .1523-47.3591$ - 181.81492 .4582 - 46.9860-183.684 92.9566 -46.3513-185.459 93.6406-45.4732 -187.082 94.2760 . . $<$ Midwall_countours $>$

$-<$ Quantitative_Information> $1.244351 .076401 .076401 .179061 .315231 .475131 .548721 .828121 .828311 .729961 .706051 .706051 .687481 .724681 .853391 .853391 .775511 . .$. 1.543431 .515551 .430361 .24435

$<$ Quantitative_Information $>$

$<$ Image_Information $>$

Fig. 4 Example of XML MIST file. A detail of geometrical and quantitative information corresponding to the selected sector of slice 3 is shown. The "midwall contours" field contains the $x, y, z$ coordinates of

integration of CTA and quantitative PET data. As previously described, each 3D visualization was rated using a five-point scale. Each observer examined the integrated data sets in random order, blinded from the results of the other observer. Agreement between obtained scores was evaluated by Wilcoxon signed-rank test. $P<0.05$ was considered statistically significant.

\section{Results}

Volumetric registration module

The method described by Marinelli et al. [18], based on the rigid transformation and on the mutual information metric each sector composing each slice of the polar map. The "quantitative information" field contains the corresponding quantitative values

[19-21] to register multimodal data sets, was used to register the reference CTA and the floating static stress and rest PET data volumes. Figure 5 shows an example of fused transaxial, coronal and sagittal views resulting from the application of the calculated registration. In all cases, the translational and rotational parameters describing the rigid transformation have been saved using the XML-transformation files.

\section{Quantitative analysis module}

The "interface" developed to save the XML MIST standard file has been integrated in a validated software for the analysis of nuclear cardiac examinations: the MunichHeart software [22]. The stress and rest dynamic PET data sets have been uploaded in the MunichHeart software and analyzed using 

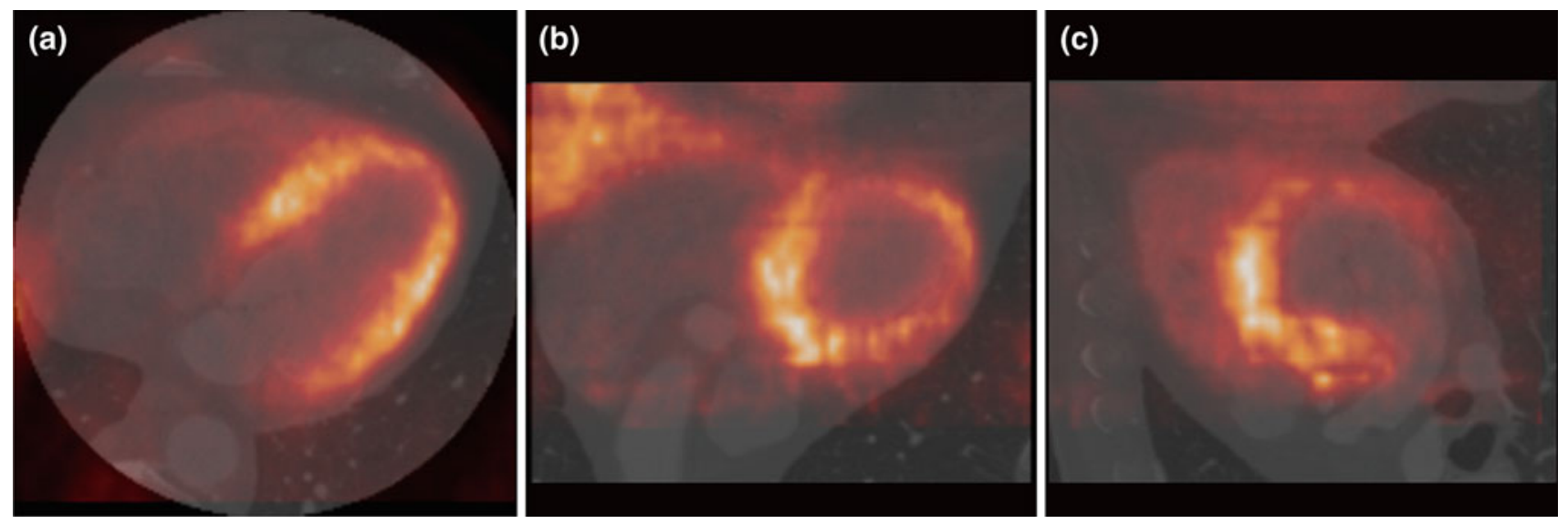

Fig. 5 Example of transaxial (a), coronal (b), and sagittal (c) views of fused CTA and original static PET

the three-compartmental model [23] for myocardial flow estimation ( $\mathrm{ml} / \mathrm{min} / \mathrm{g}$ ). Two XML MIST files corresponding to the stress and rest quantitative polar maps (15 total slices and 36 chords per slice) have been saved.

Additionally, the two XML MIST files were combined in order to calculate the myocardial flow reserve (MFR) polar map, defined as stress-to-rest ratio. The corresponding XML MIST files was also saved. Figure 6 shows the resulting stress, rest, and MFR quantitative polar maps segmented using the standard coronary artery territories AHA segmentation [9].

\section{Derived DICOM module}

The quantitative stress, rest, and MFR PET derived DICOM data sets have been built using the corresponding XML MIST files, saved following the method previously described. The fixed scale $0-3.2 \mathrm{ml} / \mathrm{min} / \mathrm{g}$ was used to visualize stress, rest, and MFR quantitative polar maps.

An example of transaxial views of the derived DICOM data sets representing the quantitative myocardial stress perfusion, myocardial rest perfusion, and calculated MFR data are shown in Fig. 7. The visual comparison between the original static PET images and the corresponding derived DICOM images is shown in Fig. 8.

To guarantee the spatial matching between the quantitative PET data and the real CTA anatomy, the corresponding header DICOM fields describing the geometrical position were further processed applying the rigid transformation saved using the XML-transformation files.

\section{$3 \mathrm{D}$ visualization}

The quantitative derived DICOM data sets representing the myocardial stress blood flow, the myocardial rest blood flow, and the MFR were uploaded to GE AWS-2.0-5.0 (Advantage Workstation ${ }^{\mathrm{TM}}$ Server). The quantitative functional data sets have been integrated to the CTA data sets representing the coronary artery anatomy using the "CardIQ Fusion" tool. An example of resulting three-dimensional fused visualizations (stress, rest, and MFR) is shown in Fig. 9.

\section{Validation}

GE AWS-2.0-5.0 server was used for fusion visualization and for $3 \mathrm{D}$ integration of anatomical and functional data sets. The proposed method was feasible when applied to all 16 data sets.

No statistically significant difference was found between the ratings provided by the two observers in all trials. In the comparison between derived and original PET data, the additional registration was performed by one observer in a single data set (average score 1.03). The mean score for the matching quality was $4.28 \pm 0.89$ (range 2:5). Matching quality was slightly higher in rest studies $(4.56 \pm 0.72)$ in respect to stress studies $(4.00 \pm 0.96)$, without reaching statistical significance $(P=0.073)$.

The mean score for matching quality in CTA/original PET data was $4.46 \pm 0.70$ (range 3:5). Matching score for CTA /derived-PET data sets was $4.31 \pm 0.83$ (range 3:5) that is not significantly different from CTA/original PET score $(P=0.275)$. Moreover, no significant difference between rest and stress data sets was assessed during CTA/PET data evaluation.

\section{Conclusion and discussion}

The CTA and quantitative PET imaging modalities have large potential in noninvasive CAD assessment giving the possibility to evaluate the presence and severity of obstructive coronary artery disease [24-27] and related quantitative information on downstream myocardial blood flow and flow reserve [12]. Moreover, three-dimensional integration of real 

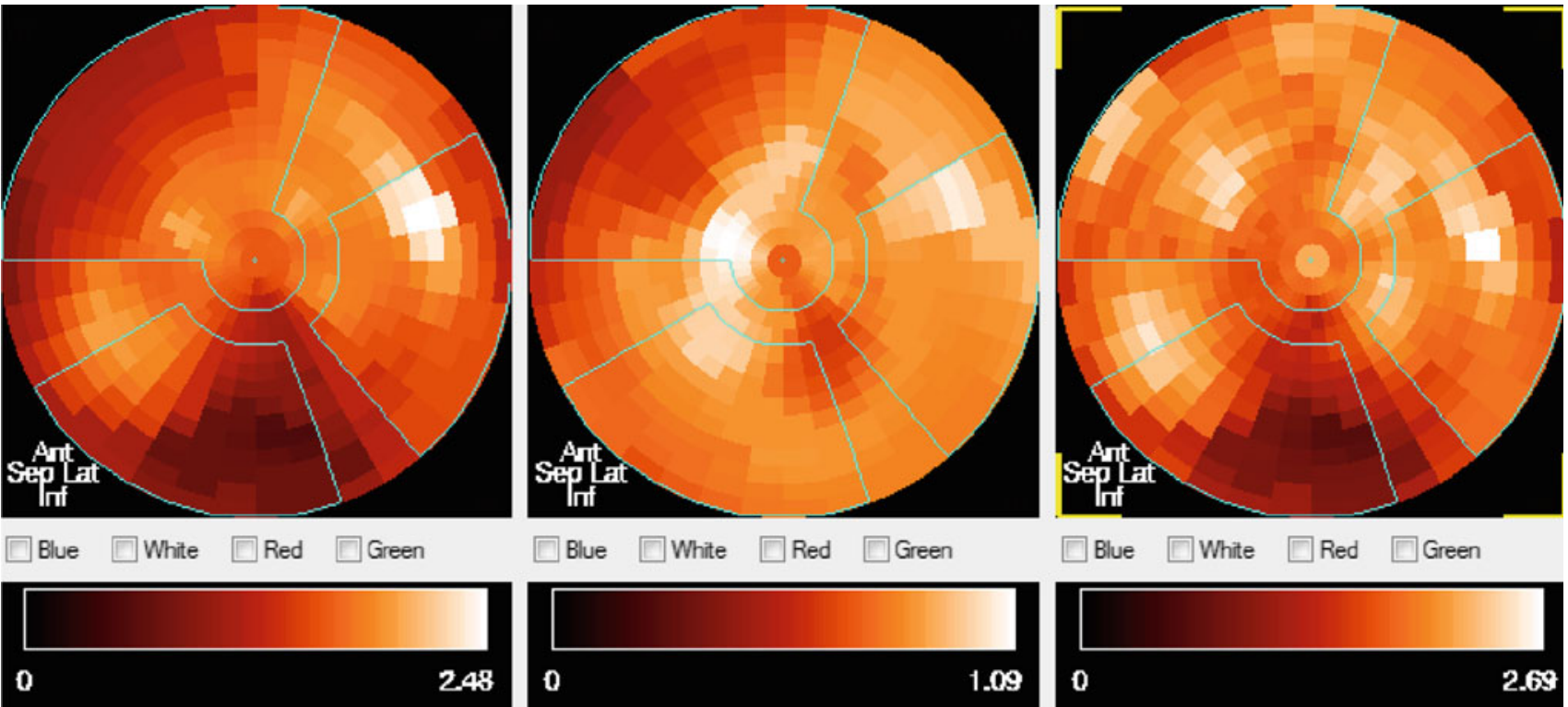

\begin{tabular}{cccl|l|}
\hline Value: & 1.48172 & & & \\
\hline ROI & \#Pts & Mean & StdDev & At \\
1ad & 160 & 1.5525 & 0.2782 & \\
rca & 80 & 1.1439 & 0.4241 \\
1cx & 80 & 1.7303 & 0.2792 & \\
\hline
\end{tabular}

(a)

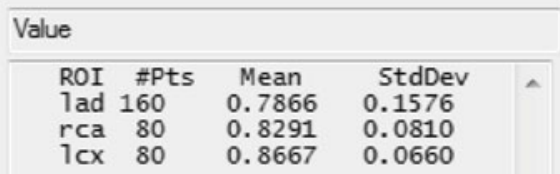

(b)

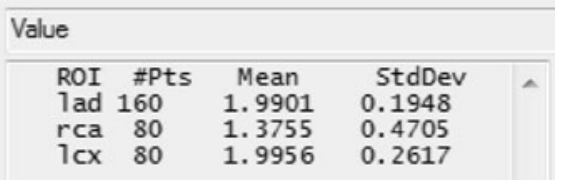

(c)

Fig. 6 Quantitative stress (a) and rest (b) and MFR (c) polar maps segmented using the standard coronary artery territories. The quantitative values are in $\mathrm{ml} / \mathrm{min} / \mathrm{g}$, and they are obtained using the analysis based on the three-compartmental model

coronary artery anatomy and quantitative myocardial blood flow can improve the assessment of cardiac diseases giving an adequate visual representation of the correspondence between anatomical pathology and their functional consequences on left ventricular perfusion.

In the clinical setting, the accomplishment of this task requires the use of two kinds of image analysis tools. Tools for PET quantification are able to infer the absolute value of blood flow from semiquantitative PET images by appropriate compartmental models and to display the myocardial blood flow values by polar maps representation. Tools for integrated 3D visualization allows to display DICOM data sets from different modalities allowing the cardiologist to interactively explore the relationship between coronary anatomy and regional myocardial perfusion. However, the use of integrated 3D visualization tools is often limited to semiquantitative PET data.

In this paper, we presented a new method to obtain an integrated 3D visualization of quantitative PET perfusion images (i.e., absolute myocardial blood flow during stress, at rest and MFR) with the corresponding coronary anatomy given by CTA imaging. Because the development of both quantitative PET images' analysis tools and integrated 3D visualization tools may require a long time and an extensive clinical validation, we preferred to integrate the existing tools in the HIT framework. As described above, the four main modules composing the developed HIT method are able to communicate by defined standard files: XML-transformation and XML MIST files. The first one has been developed to contain the information about the rigid transformation useful to align the multimodal data sets. This file allows to store and transfer the parameter transformation and to apply it to the original data set when necessary, without saving the registered DICOM data set. The proposed XML MIST standard file is the one the most relevant element composing the HIT method. It permits to store and transfer, with minimal file size, the information about the multimodal cardiac data. Indeed, it has been developed in order to contain the most relevant header DICOM information and the image information such as the geometrical position of left ventricle myocardial wall and the high resolution quantitative information. Both saved information are necessary for building the quantitative derived DICOM.

The derived DICOM data sets have the peculiarity to describe quantitative high resolution information of left ventricle myocardial perfusion. The utilization of fixed scale gives the possibility to perform an objective visual comparison of myocardial blood flow in different conditions (i.e., rest and stress). This can be useful in several clinical applications, such as during a follow-up study.

An important characteristic of the HIT process is the potential to use it in order to integrate several imaging modal- 

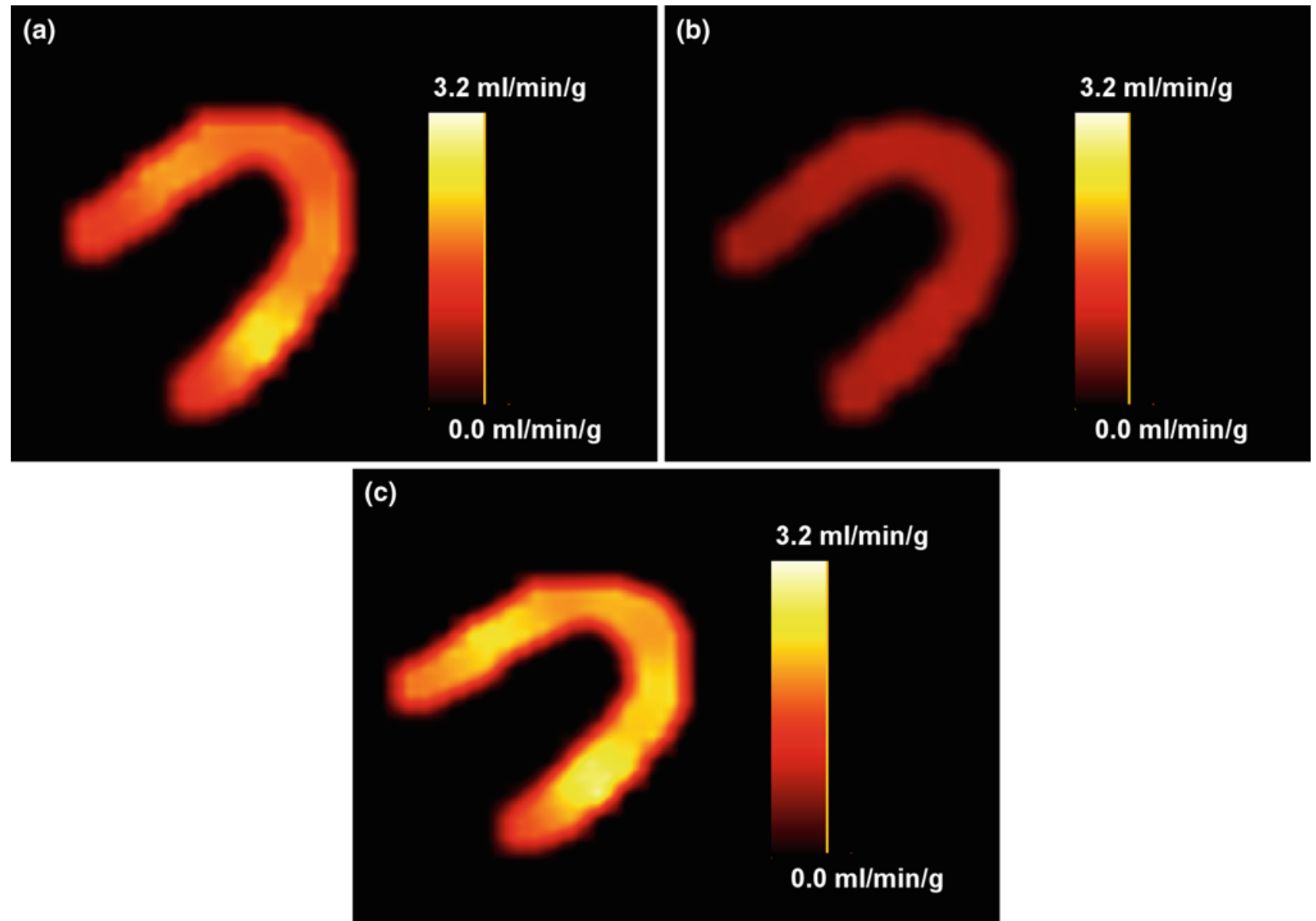

Fig. 7 Example of transaxial views of derived DICOM data sets representing the quantitative information of myocardial blood flow during stress (a), at rest (b) and MFR (c)
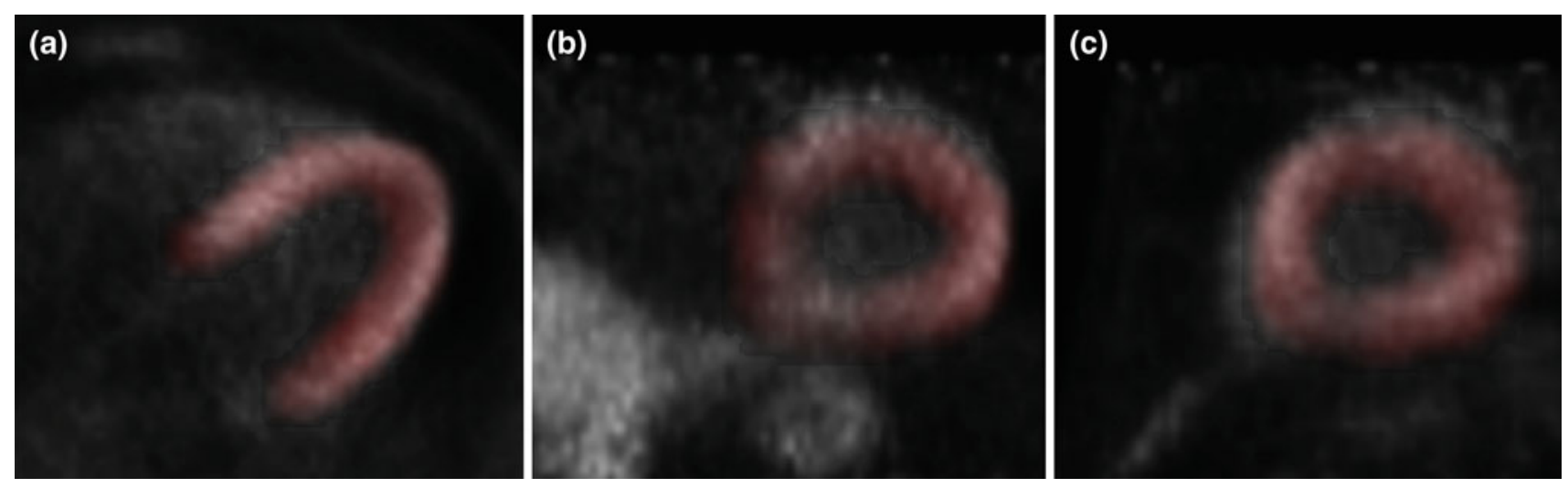

Fig. 8 Fused transaxial (a), coronal (b), and sagittal (c) views of original PET images and corresponding images resulting from the derived DICOM data set representing the quantitative myocardial perfusion

ities. Indeed, the XML-transformation file can be used as output from any registration software able to align multimodal data sets. In the same way, any software for quantitative analysis of functional cardiac data sets could be "activated" in order to save the XML MIST file.
The use of validated and/or commercial software guarantees the accuracy and reliability of the results coming from the quantitative module, avoiding the need of a dedicated development and repeated clinical validation. Obviously, it needs the integration of the developed method into 


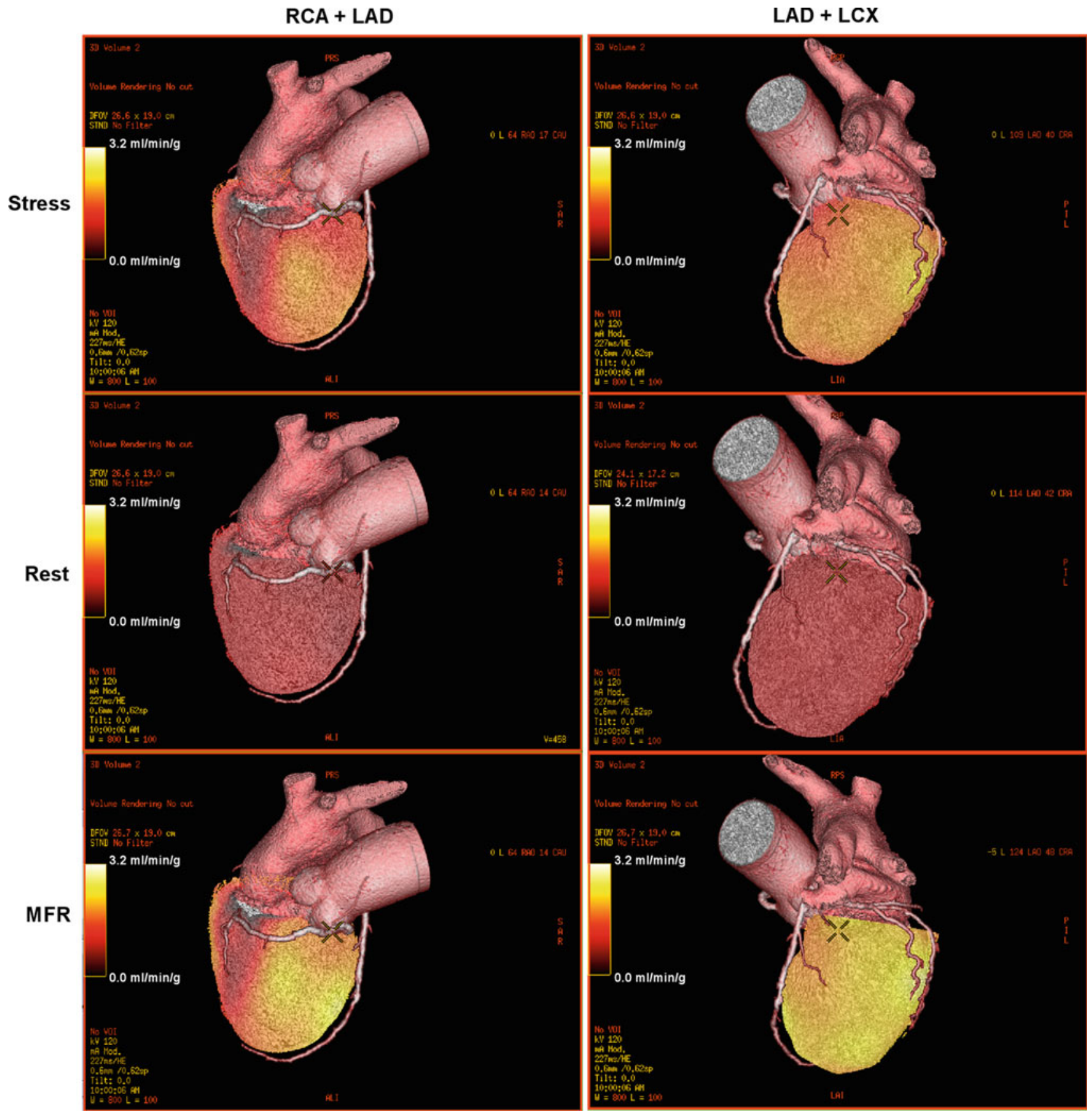

Fig. 9 Fused 3D views of quantitative PET data (stress, rest, and MFR) and coronary artery anatomy. The integrated visualization has been obtained using the "CardIQ Fusion" tool of GE AWS-2.0-5.0 (Advantage Workstation ${ }^{\mathrm{TM}}$ Server). The stress-induced regional reduc-

the "black-box" software tools by a collaboration of software owners.

The results of the validations carried out in the study confirmed the high matching between original and derived data sets and also the good quality of the registration procedure that is fully comparable in original and derived data. tion in hyperemic flows, subtended to a flow-limiting epicardial lesion, is observable in the territory of right coronary artery. This result has been confirmed by invasive coronary angiography examination

Indeed, a key point in the HIT is the accurate spatial matching between data sets involved in it. For this reason, a registration algorithm has been applied to match the stress and rest PET to CTA images (Fig. 3, module 1) permitting to have stress, rest, and derived quantitative data sets registered to CTA anatomy. 
This registration algorithm is based on the mutual information metric that is voxel-based method and does not make any assumptions about the relationship between various image intensities [19-21]. For this reason, it has the advantage to be applied for the alignment of multimodal cardiac imaging. In all of the cases, the rigid transformation, described by three translational and three rotational parameters, has been exploited due to its wide use in commercial clinical software to align PET and CTA data sets. The eventual application of the nonrigid registration would require the adjustment of XML-transformation file to the specific purpose. From the saved XML files, the quantitative derived DICOM data set can be created and imported in any software for multimodal cardiac visualization and fusion. In this paper, derived DICOM data sets representing quantitative myocardial blood flow have been created, but further complementary and quantitative data can be considered. This has been demonstrated in a preliminary study integrating CTA anatomy and quantitative myocardial motion assessed using MR imaging [28]. Additionally, other data could be potentially integrated such as the viability information evaluated by ${ }^{18}$ FDG-PET [29] or by delayed enhancement of MR imaging [30] and the myocardial contractility assessed using tagging MR imaging [31].

In conclusion, in this paper, we presented a new method to integrate and three dimensionally visualize coronary artery anatomy obtained by CTA imaging and quantitative myocardial blood flow resulting from PET imaging. This new technique can provide significant improvement in clinical assessment of cardiac diseases.

Acknowledgments The research leading to these results has received funding from the European Community's Seventh Framework Programme (FP7/2007-2013) under grant agreement no. 222915 (EVINCI study). EVINCI study is also supported by GE Healthcare.

Conflict of interest None.

\section{References}

1. Di Carli MF, Dorbala S, Hachamovitch R (2006) Integrated cardiac PET-CT for the diagnosis and management of CAD. J Nucl Cardiol 13:139-144

2. Gaemperli O, Kaufmann PA (2008) Hybrid cardiac imaging: more than the sum of its parts. J Nucl Cardiol 15:123-126

3. Di Carli MF, Hachamovitch R (2008) Hybrid PET/CT is greater than the sum of its parts. J Nucl Cardiol 15:118-122

4. Javadi MS, Lautamäki R, Merrill J, Voicu C, Epley W, McBride G, Bengel FM (2010) Definition of vascular territories on myocardial perfusion images by integration with true coronary anatomy: a hybrid PET/CT analysis. J Nucl Med 51:198-203

5. Knuuti J, Kaufmann PA (2009) Hybrid imaging: PET-CT and SPECT-CT. In: Zamorano JL et al (eds) The ESC textbook of cardiovascular imaging. Springer, London, Limited 2010, pp 89-99

6. Beller GA (2010) Recent advances and future trends in multimodality cardiac imaging. Heart Lung Circ 19:193-209
7. Marinelli M, Positano V, Tucci F, Neglia D (2012) Automatic hboxPET-CT image registration method based on mutual information and genetic algorithms. The Scientific World Journal, vol 2012, Article ID 567067, p 12. doi:10.1100/2012/567067

8. Mäkelä T, Clarysse P, Sipilä O, Pauna N, Pham QC, Katila T, Magnin IE (2002) A review of cardiac image registration methods. IEEE Trans Med Imag 21:1011-1021

9. Cerqueira MD, Weissman NJ, Dilsizina V, Jacobs AK, Kaul S, Laskey WK, Pennell DJ, Rumberger JA, Ryan T, Verani MS (2002) Standardized myocardial segmentation and nomenclature for tomographic imaging of the heart: a statement for healthcare professionals from the cardiac imaging committee of the council on clinical cardiology of the american heart association. Circulation 105:539-542

10. Masci PG, Marinelli M, Piacenti M, Lorenzoni V, Positano V, Lombardi M, L'Abbate A, Neglia D (2010) Myocardial structural, perfusion and metabolic correlates of left bundle branch block mechanical derangement in patients with dilated cardiomyopathy-a tagged cardiac magnetic resonance and positron emission tomography study. Circ Cardiovasc Imaging 3:482-490

11. Kaufmann PA, Camici PG (2004) Myocardial blood flow measurement by PET: technical aspects and clinical application. J Nucl Med 46:75-88

12. Schindler TH, Schelbert HR, Quercioli A, Dilsizian V (2010) Cardiac PET imaging for detection and monitoring of coronary artery disease and microvascular health. JACC Imaging 3:623-640

13. Stolzmann P, Alkadhi H, Scheffel H, Hennemuth A, Kuehnel C, Baumueller S, Kozerke S, Falk V, Marincek B, Donati OF (2010) Image fusion of coronary CT angiography and cardiac perfusion MRI: a pilot study. Eur Radiol 20:1174-1179

14. Kajander S, Joutsiniemi E, Saraste M, Pietilä M, Ukkonen H, Saraste A, Sipilä HT, Teräs M, Mäki M, Airaksinen J, Hartiala J, Knuuti J (2010) Cardiac positron emission tomography/computed tomography imaging accurately detects anatomically and functionally significant coronary artery disease. Circulation 122:603613

15. Knuuti J, Kajander S, Mäki M, Ukkonen H (2009) Quantification of myocardial blood flow will reform the detection of CAD. J Nulc Cardiol 16:497-506

16. Neglia D, Michelassi C, Trivieri MG, Sambuceti G, Giorgetti A, Pratali L, Gallopin M, Salvadori P, Sorace O, Carpeggiani C, Poddighe R, L'Abbate A, Parodi O (2002) Prognostic role of myocardial blood flow impairment in idiopathic left ventricular dysfunction. Circulation 105:186-193

17. Extensible Markup Language (XML) 1.0 (Fifth Edition) W3C Recommendation 26 Nov 2008. Available: http://www.w3.org

18. Marinelli M, Martinez-Möller A, Jensen B, Positano V, Weissmüller S, Navab N, Landini L, Schwaiger M, Nekolla SG (2010) Registration of myocardial PET and SPECT for viability assessment using mutual information. Med Phys 37:2414-2424

19. Pluim JPW, Maintz JBA, Viergever MA (2003) Mutual-information-based registration of medical images: a survey. IEEE Trans Med Imaging 22:986-1004

20. Viola P, Wells WM (1997) Alignment by maximization of mutual information. Int J Comput Vis 24:137-154

21. Collignon A, Vandermeulen D, Suetens P, Marchal G (1995) 3D multimodality medical image registration using feature space clustering. In: Ayache N (ed) Proceedings of the CVRMed lecture notes in computer science 905/1995. Springer, Berlin, pp 193-204

22. Nekolla SG, Miethaner C, Nguyen N, Ziegler SI, Schwaiger M (1998) Reproducibility of polar map generation and assessment of defect severity and extent assessment in myocardial perfusion imaging using positron emission tomography. Eur J Nucl Med Mol Imaging 25:1313-1321

23. Musik O, Beanlands RSB, Hutchins GD, Mangner TJ, Nguyen N, Schwaiger M (1993) Validation of nitrogen-13-ammonia tracer 
kinetic model for quantification of myocardial blood flow using PET. J Nucl Med 34:83-91

24. Miller JM et al (2008) Diagnostic performance of coronary angiography by 64-row CT. N Engl J Med 359:2324-2336

25. Meijboom WB et al (2008) Diagnostic accuracy of 64-slice computed tomography coronary angriography. A prospective, multicenter, multivendor study. J Am Coll Cardiol 52:2135-2144

26. Budoff MJ et al (2008) Diagnostic performance of 64-multidetector row coronary computed tomographic angiography for evaluation of coronary artery stenosis in individuals withouit known coronary artery disease. J Am Coll Cardiol 52:1724-1732

27. Miny JK et al (2011) Age- and sex-related differences in all-cause mortality risk based on coronary computer tomography angiography findings. J Am Coll Cardiol 58:849-860
28. Marinelli M, Positano V, Marcheschi P, Todiere G, Esposito N, Puzzuoli S, Marraccini P, Lombardi L, Landini L, Neglia D (2011) Integrated visualization of coronary anatomy and quantitative MRI by CT-MRI image fusion. Magn Reson Mater Phy 24(Supp.1):406

29. Segall G (2002) Assessment of myocardial viability by positron emission tomography. Nucl Med Commun 23:323-330

30. Saraste A, Nekolla SG, Schwaiger M (2008) Contrast-enhanced magnetic imaging in the assessment of myocardial infarction and viability. J Nucl Cardiol 15:105-117

31. Axel L, Montillo A, Kim D (2005) Tagged magnetic resonance imaging of the heart: a survey. Med Image Anal 9:376-393 